\title{
A DETAILED STUDY OF THE GALACTIC PLANETARY NEBULA
}

G 258-15.7

\author{
P. LEISY and M. DENNEFELD \\ Institut d'Astrophysique de Paris, IAP/CNRS
}

The galactic Planetary Nebula G 258-15.7 is a large, bright nebula well suited for a detailed study. Known since Wray (1966), its morphology presents several blobs and ansae, generally associated with type I nebulae, and could be described as "late-butterfly" type according to the classification by Balick (1989). The central star has been classified as hydrogen-deficient by Mendez et al. (1985). Spectroscopy of the two main blobs shows a clear overabundance in He and $\mathrm{N}$, with a marginally significant difference between the two sides. The most striking feature is the jet-like structure appearing on the [OIII]/Halpha picture (Fig. 1), the "jets" being located within the main blobs seen on the monochromatic images. A detailed appraisal of all the data will be presented in a subsequent paper.

\section{References}

Balick, B., 1989, in IAU Symp. 131, p. 83, S. Torres-Peimbert, Edt

Mendez, R.H., Kudritzki, R.P. and Simon, K.P., 1985, Astron. Astrophys. 142, 289

Wray $_{5}$ J.D., 1966, Ph.D Thesis, Northwestern University

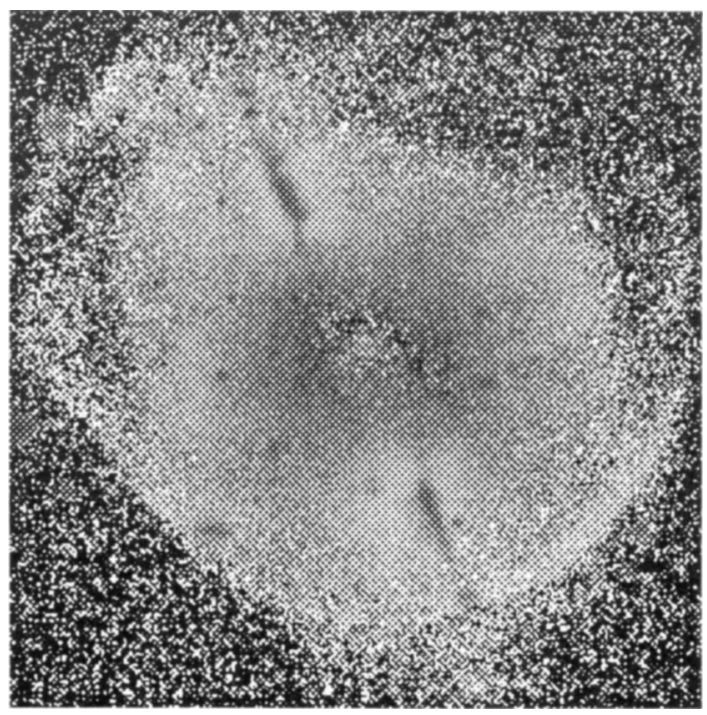

Fig. 1. $\underline{\mathrm{NW} \text { blob }}: \mathrm{He} / \mathrm{H}=0.15 ; \mathrm{N} / \mathrm{O}=0.27 . \underline{\mathrm{SE} \text { blob }}: \mathrm{He} / \mathrm{H}=0.14 ; \mathrm{N} / \mathrm{O}=0.21$. 